\section{Evaluation of pain during intravitreal Ozurdex injections vs intravitreal bevacizumab injections}

E Moisseiev, M Regenbogen, T Rabinovitch, A Barak, A Loewenstein and M Goldstein
Department of

Ophthalmology, Tel Aviv Sourasky Medical Center, Affiliated to the Sackler Faculty of Medicine, Tel Aviv University, Tel Aviv, Israel

Correspondence:

E Moisseiev, Department of Ophthalmology, Tel Aviv Sourasky Medical Center, Weitzman 6 Street, Tel Aviv 64239 Israel

Tel: +972 36973408 ;

Fax: +97236973870

E-mail: elad_moi@

netvision.net.il

Received: 24 October 2013 Accepted in revised form:

11 May 2014

Published online:

13 June 2014

\section{Abstract}

Purpose The purpose was to evaluate the pain associated with intravitreal Ozurdex injections, and to compare it with that associated with intravitreal bevacizumab injections.

Methods The study included 57 eyes of 57 patients who received an intravitreal Ozurdex injection at our institution. Pain was measured by the visual analog scale (VAS). Additional parameters recorded included age, sex, indication for the injection, number of previous Ozurdex injections in the study eye, presence of diabetes mellitus, and lens status. Data were compared with a 2:1 sexand age-matched control group of 114 patients who received intravitreal bevacizumab injections.

Results Indications for injection included diabetic macular edema (40.4\%) and macular edema secondary to central and branch retinal vein occlusion $(28 \%$ and $31.6 \%$, respectively). Pain scores on the VAS ranged from 0 to 90 , with a mean of $20.8 \pm 20.3$. There was no significant difference in pain between Ozurdex and bevacizumab injections. Pseudophakia was correlated with increased pain in Ozurdex injections. Conclusions This is the first series evaluating the pain associated with intravitreal Ozurdex injections. Despite a larger needle gauge and tunneled injection technique, intravitreal injection of Ozurdex is not associated with increased pain compared with bevacizumab. This finding may be a potential advantage for Ozurdex, and may serve to improve patient compliance with future long-term treatment protocols.

Eye (2014) 28, 980-985; doi:10.1038/eye.2014.129; published online 13 June 2014
Introduction

Ozurdex (Allergan Inc., Irvine, CA, USA) is a biodegradable, slow-release dexamethasone implant delivered by intravitreal injection. It is composed of a biodegradable copolymer matrix of lactic acid and glycolic acid that enables the slow release of dexamethasone. ${ }^{1}$ Ozurdex has been studied mainly for the treatment of macular edema (ME) associated with retinal vascular occlusion and noninfectious posterior uveitis, ${ }^{1-6}$ and has been approved by the regulatory agencies in the United States and Europe for these indications. Ozurdex was also shown to be effective in the treatment of diabetic macular edema (DME) in smaller studies, ${ }^{7-10}$ and several other studies have reported its efficacy in treating ME secondary to numerous other diseases, including Irvine-Gass syndrome ${ }^{11,12}$ Coat's disease, $^{13}$ radiation retinopathy, ${ }^{14}$ and cystoid macular edema (CME) secondary to retinitis pigmentosa (RP). ${ }^{15}$

Ozurdex has a favorable safety profile, and its most commonly recorded complication is a transient elevation in intraocular pressure (IOP) that is usually managed by topical medication. ${ }^{2,16}$ In light of the growing body of evidence demonstrating that Ozurdex is a safe and efficient treatment modality for a variety of indications, it is expected that its use in clinical practice and clinical trials will increase in the future.

In the CHAMPLAIN study, a phase 2 study that evaluated the efficacy of Ozurdex for the treatment of DME in vitrectomized eyes, eye pain during the injection was reported by $16 \%$ of patients, and was considered a common adverse effect of this injection. ${ }^{7}$ Pain was only reported by the patients and not quantified in any way. 
None of the other studies using Ozurdex had investigated the pain that patients experience during the injection. The purpose of this study was to evaluate and quantify pain during Ozurdex injection, and to determine whether any of the patients' parameters are in correlation with it. A comparison between pain estimation during intravitreal injections of Ozurdex and bevacizumab (Genentech/ Roche, San Francisco, CA, USA) was also performed.

\section{Materials and methods}

\section{Patient selection}

The study was approved by our institutional review board, and all patients gave written informed consent before their participation. All patients included in this study received intravitreal Ozurdex injections in our clinic between 1 April 2012 and 31 March 2013. All patients were $\geq 18$ years old and were referred to the injection by retina specialists from our institution. Indications for injection included $\mathrm{ME}$ secondary to central retinal vein occlusion (CRVO), branch retinal vein occlusion (BRVO), or diabetic retinopathy (DR).

Exclusion criteria included any previous ocular surgery other than cataract extraction. All pseudophakic patients included in the study had a posterior chamber intraocular lens (IOLs) placed in the capsular bag. Patients with anterior chamber IOLs, sulcus placed IOLs, or aphakia were excluded. Patients with anterior segment conditions that could affect pain sensation, such as conjunctival irritation, active conjunctivitis or keratitis or bullous keratopathy, were also excluded. Patients using systemic analgesic or sedative medications were excluded.

The group of patients who received Ozurdex injections was compared with an age- and sex-matched control group who received intravitreal bevacizumab injections. Patients in the control group were selected from among a cohort of 218 patients who participated in a previous study by our group. ${ }^{17}$ A 2:1 sampling ratio was used, matching two patients who received an intravitreal bevacizumab injection with each patient who received Ozurdex.

\section{Data collection}

Each participant received a single Ozurdex injection in one eye during the study. Additional parameters recorded for the participants included age, sex, the underlying retinal cause for the injection, number of previous Ozurdex injections in the injected eye, presence of diabetes mellitus (DM), lens status, and the pain associated with the injection. 'Injection number' was defined as the number of previous intravitreal injections in the eye plus one (eg, 1 for an eye injected for the first time, and 5 for an eye that received 4 injections before the study).

Pain was measured by subjective grading on a visual analog scale (VAS). The VAS is a horizontal line measuring exactly $10 \mathrm{~cm}(100 \mathrm{~mm})$, as shown in Figure 1. All VAS measurements were collected by a single ophthalmologist (EM) after explaining this method to the patients. Each patient was asked to mark a vertical line crossing the horizontal line, according to his or her subjective pain assessment during the injection, ranging from no pain at all to maximal pain. The distance between the left edge of the horizontal line and the vertical mark made by the patient was later measured and recorded in $\mathrm{mm}$, and transformed into a score between 0 and 100. The VAS is a common tool for assessing pain and other symptoms, and has been shown to be a valid and reliable research method in previous clinical studies. ${ }^{18-21}$ It has also been successfully used in ophthalmological studies evaluating pain associated with ocular surgery, intravitreal injections, and topical therapies. ${ }^{17,22-28}$ The patients received an explanation of the VAS before the injection, and reported immediately following the procedure the degree of pain they felt during the injection.

\section{Intravitreal injection technique}

Anesthetic technique was uniform for all participants, and included $1 \mathrm{ml}$ of $2 \%$ lignocaine gel (Esracaine Jelly, Rafa Laboratories LTD, Jerusalem, Israel) 15 and $5 \mathrm{~min}$ before injection. No sedation was administered to any patient. The same topical anesthetic technique was also used in our previous study evaluating pain during intravitreal bevacizumab injection, whose participants were included in the control group.

All intravitreal injections were performed in the supine position under sterile conditions. The eyelids were washed three times using a $4 \%$ povidone-iodine solution and then dried with sterile gauze. A sterile plastic drape

\section{Please make a mark on the line according to the pain you felt during the Ozurdex injection:}

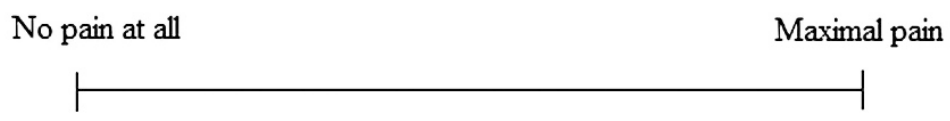

Figure 1 The visual analog scale used in the study. The line is exactly $100 \mathrm{~mm}$ long. 
was placed on the eyelashes in order to shift them away from the ocular surface. A sterile speculum was placed to make sure the eyelids remain open throughout the procedure. Then, $4 \%$ povidone-iodine was applied to the conjunctival and corneal surface. Calipers were used to mark the injection site, $3.5 \mathrm{~mm}$ from the limbus, where the injection of a $700 \mu \mathrm{g}$ dexamethasone implant (Ozurdex) was made. All injections were performed using a tunneled injection technique, creating a 'bent' intrascleral route. All injections were made using the customized applicator that has a 22-gauge needle. ${ }^{1}$ After removing the applicator, mild pressure was applied with a cotton swab over the injection site to reduce vitreal reflux and subconjunctival hemorrhage. All patients received a drop of antibiotic (Ciprofloxacin; Ciloxan, Luxemburg Pharmaceuticals, Tel Aviv, Israel) immediately after the intravitreal injection.

\section{Study power calculation}

When designing this study, an a priori power calculation was performed to determine the required sample sizes for the Ozurdex and control groups. In our previous study on pain associated with intravitreal bevacizumab injections, we found the mean and SD of the VAS score to be $17.4 \pm 17.1{ }^{17}$ Assuming that a difference of 10 in the VAS score was of clinical significance, it was calculated that for an Ozurdex group of 50 patients and a control group of 100 patients, the study power would be $92.1 \%$.

\section{Statistical analysis}

Demographic and patient characteristics were compared using descriptive statistics and univariate analysis as appropriate. Because of skewed distributions of VAS scores, categorical parameters were analyzed using Mann-Whitney test and continuous parameters were analyzed by Spearman's rank correlations. Analysis of covariance (ANCOVA) was also used to analyze the correlation between pain and patient age and lens status. The statistical significance level was set at 0.05 in all statistical analyses, including the $a$ priori power calculation. Data were analyzed using SPSS for Windows, version 18.0 (SPSS Inc., Chicago, IL, USA).

\section{Results}

A total of 57 eyes of 57 patients who met the inclusion criteria were included in the study. There were $36(63.2 \%)$ men and $21(36.8 \%)$ women, with a mean age of $65.7 \pm 13.1$ years (range 26 to 92 years). Of these, 39 (68.4\%) eyes were phakic and 18 (31.6\%) were pseudophakic at the time of Ozurdex injection. Presence of DM was recorded in $27(47.4 \%)$ patients. Indications for Ozurdex injection included DME (23 patients, 40.4\%) and ME secondary to CRVO (16 patients, 28\%) and BRVO (18 patients, 31.6\%). Injection number ranged from 1 to 5 , with a mean of $1.68 \pm 0.92$ injections. Of the eyes, 31 (54.4\%) included in the study were injected with Ozurdex for the first time. No adverse events other than mild transient subconjunctival hemorrhage were encountered during the study.

Pain scores on the VAS ranged from 0 to 90 , with a mean of $20.8 \pm 20.3$. No correlation was found between pain scores and patient sex, indication for Ozurdex injection, or presence of DM. No correlation was demonstrated between pain scores and number of injection. There was also no difference in pain scores between eyes injected for the first time and eyes that were previously injected.

Patient age was significantly correlated with higher VAS pain scores $(P=0.017)$. Pseudophakia was also significantly correlated with higher pain scores $(P=0.005)$. Mean VAS pain scores were $32.3 \pm 23.0$ in pseudophakic eyes and 15.6 \pm 16.8 in phakic eyes (Figure 2). ANCOVA was performed using square-root transformation of the VAS score to normalize their distribution. In this analysis, only pseudophakia was demonstrated to be significantly correlated with pain $(P=0.005)$.

\section{Comparison of pain between intravitreal Ozurdex and bevacizumab injections}

A 2:1 sex- and age-matched control group of patients who reported their pain during bevacizumab injection on VAS was designed. This group consisted of 114 patients, of whom $58(50.9 \%)$ were treated for neovascular agerelated macular degeneration (AMD), 40 (35.1\%) for

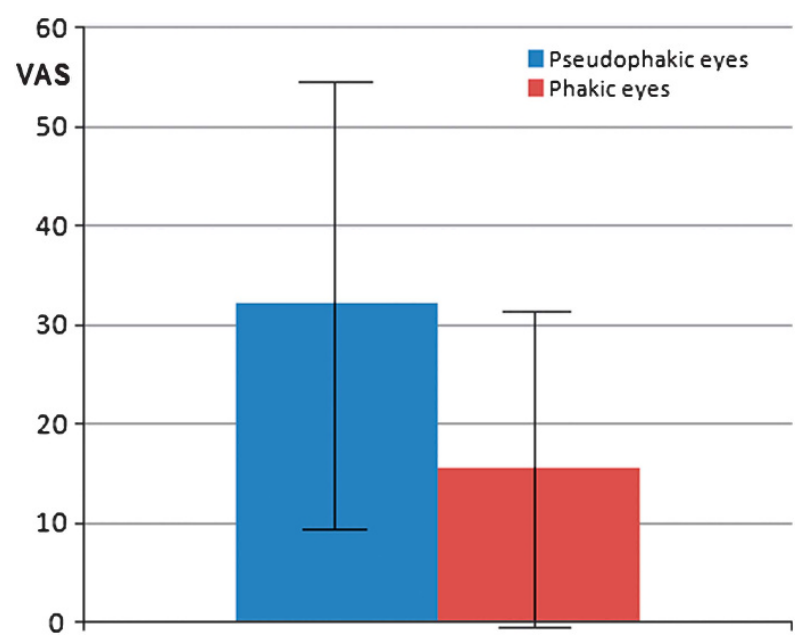

Figure 2 Comparison of mean and SD of VAS pain scores between phakic and pseudophakic patients. The difference was statistically significant $(P=0.005)$. 
DME, and 16 (14\%) for ME secondary to CRVO or BRVO. There were no statistically significant differences between the Ozurdex and the control group (Table 1).

In the control group, none of the recorded parameters was correlated with pain.

Mean VAS pain scores were $20.8 \pm 20.3$ in the Ozurdex group and $16.6 \pm 17.4$ in the control group. No significant difference was demonstrated between the two groups regarding pain during the injection $(P=0.151)$. To compensate for the differences in indications for injections, subanalyses were made comparing patients who were treated for similar indications. No significant differences in VAS pain scores were found when comparing Ozurdex and bevacizumab injections in patients with DME $(P=0.837)$ and in patients with ME secondary to CRVO or BRVO $(P=0.304)$.

\section{Discussion}

Intravitreal injections have become integral in retinal practice, and it is estimated that in the near future they are likely to become the most common intraocular procedure performed worldwide. ${ }^{29}$ As the majority of patients require multiple injections, evaluating the pain associated with this procedure is important to optimize patient comfort and compliance.

Previous published studies on pain associated with intravitreal injections included only bevacizumab or ranibizumab injections. Most of these studies were designed to evaluate the efficacy of different anesthetic protocols. Although no consensus has been reached, topical anesthesia by either drops or gel has been repeatedly shown to be very effective in pain control during intravitreal injections. ${ }^{26-28,30-33}$ Therefore, it has been generally agreed that topical anesthesia is most comfortable and cost effective,,$^{27,32,34}$ and this is currently the most commonly used protocol before intravitreal injections. Pain associated with intravitreal injections is generally mild, and mean VAS scores reported in previous studies during these injections varied from 12 to 35. ${ }^{17,30,31,35-38}$ A previous study performed in our department regarding pain sensation associated with

Table 1 Comparison of the Ozurdex group and the bevacizumab control group

\begin{tabular}{lccc}
\hline Parameter & $\begin{array}{c}\text { Ozurdex } \\
(\mathrm{N}=57)\end{array}$ & $\begin{array}{c}\text { Bevacizumab controls } \\
(\mathrm{N}=114)\end{array}$ & P-value \\
\hline Age (years) & $65.7 \pm 13.1$ & $68.8 \pm 9.2$ & 0.112 \\
Male patients (\%) & $63.2 \%$ & $59.6 \%$ & 0.740 \\
Diabetes mellitus (\%) & $47.4 \%$ & $43 \%$ & 0.626 \\
Pseudophakia (\%) & $31.6 \%$ & $24.6 \%$ & 0.363 \\
VAS pain score & $20.8 \pm 20.3$ & $16.6 \pm 17.4$ & 0.151 \\
\hline
\end{tabular}

No significant differences existed between the groups. intravitreal injections of bevacizumab includes the largest series (218 patients) published to date. ${ }^{17}$ No correlation was found between pain and age, sex, indication for injection, number of previous injections, presence of DM, and lens status. The scleral location of the intravitreal injection was not correlated with pain as well, but a trend toward milder pain was noted when the physician performed the injection at the quadrant he was most accustomed to. ${ }^{17}$

Our results demonstrated no significant difference in pain between intravitreal injection of Ozurdex and bevacizumab. There are two important differences between these two intravitreal injections. First, Ozurdex was injected using a 22-gauge needle that is 2.25 times wider than the 29-gauge needle used for bevacizumab injection ( 0.644 and $0.286 \mathrm{~mm}$, respectively). Second, Ozurdex injection is performed through a tunneled injection tract in the sclera, whereas bevacizumab injection is performed through a straight penetration. It has been previously demonstrated that smaller gauge needles are associated with less pain in intravitreal injection. ${ }^{37}$ Tunneled injection was not shown to be associated with increased pain compared with straight injection. ${ }^{37,38}$ Ozurdex injection requires a tunneled technique to prevent vitreal reflux through the 22-gauge scleral puncture. Our results demonstrate that this wider and slightly longer intrascleral injection tract is not associated with increased pain during this procedure. We propose two possible explanations for this finding. First, the similarity in pain may reflect the use of the same topical anesthesia protocol in both groups. Topical anesthesia with lignocaine gel is very effective for intravitreal injections, ${ }^{30-34}$ and it may have blunted the increased pain that could have resulted from the larger Ozurdex injection. Second, Ozurdex was injected through a customized applicator, whereas bevacizumab was injected from a simple syringe. The design of the applicator could perhaps be responsible for a lower level of pain during the injection that may compensate for its wider size.

This is the first study to evaluate pain associated with intravitreal injections of Ozurdex. No correlation was found between pain scores and patient sex, indication for injection, number of previous injections, or presence of DM. A surprising finding was the significant correlation of increased pain with pseudophakia (Figure 2). This correlation has never been reported in previous studies on intravitreal injections, and was not demonstrated in our large series of bevacizumab injections ${ }^{17}$ or in the control group in this study. Although statistically significant, we do not believe this finding is clinically important.

A potential limitation of this study is the fact that Ozurdex injections were not administered by a single 
ophthalmologist. However, all injections were performed by specialists who had previous experience in Ozurdex injections. The series size and 2:1 sex- and age-matched control group are methodological strengths of this study.

In conclusion, this is the first comprehensive evaluation of pain associated with Ozurdex intravitreal injections. The degree of pain associated with Ozurdex injections was not significantly different than that reported for intravitreal bevacizumab injections in a matched control group. An understanding of the pain associated with Ozurdex injections may be important in these considerations, and may have a role in improving patient compliance.

\section{Summary}

\section{What was known before}

- No previous studies focused on pain during intravitreal Ozurdex injection.

- Ocular pain during Ozurdex injection is common ( $16 \%$ of patients report it).

What this study adds

- Intravitreal injection of Ozurdex is not associated with more pain than bevacizumab injections, despite larger needle gauge and longer scleral route.

- Pseudophakia is associated with higher pain scores reported during Ozurdex injection.

- Patient age, indication for injection, and number of injection do not correlate with the level of pain.

\section{Conflict of interest}

The authors declare no conflict of interest.

\section{References}

1 Haller JA, Bandello F, Belfort R, Blumenkranz MS, Gillies M, Heier $\mathrm{J}$ et al. Randomized, sham-controlled trial of dexamethasone intravitreal implant in patients with macular edema due to retinal vein occlusion. Ophthalmology 2010; 117: 1134-1146.

2 Haller JA, Bandello F, Belfort R, Blumenkranz MS, Gillies M, Heier J et al. Dexamethasone intravitreal implant in patients with macular edema related to branch or central retinal vein occlusion. Ophthalmology 2011; 118: 2453-2460.

3 Herrero-Vanrell R, Cardillo JA, Kuppermann BD. Clinical applications of the sustained-release dexamethasone implant for the treatment of macular edema. Clin Ophthalmol 2011; 5: 139-146.

4 Lowder C, Belfort Jr R, Lightman S, Foster CS, Robinson MR, Schiffman RM et al. Dexamethasone intravitreal implant for noninfectious intermediate or posterior uveitis. Arch Ophthalmol 2011; 129: 545-553.

5 Mayer WJ, Wolf A, Kernt M, Kook D, Kampik A, Ulbig M et al. Twelve-month experience with Ozurdex for the treatment of macular edema associated with retinal vein occlusion. Eye 2013; 27: 816-822
6 Bezatis A, Spital G, Hohn F, Maier M, Clemens CR, Wachtlin $\mathrm{J}$ et al. Functional and anatomical results after a single intravitreal Ozurdex injection in retinal vein occlusion: a 6 month follow-up-The SOLO study. Acta Ophthalmol 2013; 91: e340-e347.

7 Boyer DS, Faber D, Gupta S, Patel SS, Tabandeh H, Li XYet al. Dexamethasone intravitreal implant for treatment of diabetic macular edema in vitrectomized patients. Retina 2011; 31: 915-923.

8 Zucchiatti I, Lattanzio R, Querques G, Querques L, Del Turco C, Cascavilla ML et al. Intravitreal dexamethasone implant in patients with persistent diabetic macular edema. Ophthalmologica 2012; 228: 117-122.

9 Matonti F, Hoffart L, Baeteman C, Denis D. Repeated treatment for macular edema in vein occlusion by intravitreal implant of dexamethasone. Case Rep Ophthalmol 2012; 3: 339-342

10 Callanan DG, Gupta S, Boyer DS, Ciulla TA, Singer MA, Kuppermann BD et al. Dexamethasone intravitreal implant in combination with laser photocoagulation for the treatment of diffuse diabetic macular edema. Ophthalmology 2013; 120(9): 1843-1851.

11 Meyer LM, Schonfeld CL. Cysoid macular edema after complicated cataract surgery resolved by an intravitreal dexamethasone 0.7-mg implant. Case Rep Ophthalmol 2011; 2: 319-322.

12 Dutra Medeiros M, Navarro R, Garcia-Arumi J, Mateo C, Corcostegui B. Dexamethasone intravitreal implant for treatment of patients with recalcitrant macular edema resulting from Irvine-Gass syndrome. Invest Ophthalmol Vis Sci 2013; 54: 3320-3324.

13 Martinez-Castillo S, Gallego-Pinazo R, Dolz-Marco R, Marin-Lambies C, Diaz-Llopus M. Adult coat's disease successfully managed with the dexamethasone intravitreal implant (Ozurdex) combined with retinal photocoagulation. Case Rep Ophthalmol 2012; 3: 123-127.

14 Russo A, Avitabile T, Uva M, Faro S, Franco L, Sanfilippo M et al. Radiation macular edema after Ru-106 plaque brachytherapy for choroidal melanoma resolved by an intravitreal dexamethasone $0.7-\mathrm{mg}$ implant. Case Rep Ophthalmol 2012; 3: 71-76.

15 Srour M, Querques G, Leveziel N, Zerbib J, Tilleul J, Boulanger-Scemama $\mathrm{E}$ et al. Intravitreal dexamethasone implant (Ozurdex) for macular edema secondary to retinitis pigmentosa. Graefes Arch Clin Exp Ophthalmol 2013; 251: 1501-1506.

16 Schmitz K, Maier M, Clemens CR, Hohn F, Watchtlin J, Lehmann $\mathrm{F}$ et al. Reliability and safety of intravitreal Ozurdex injections: the ZERO study. Ophthalmologue 2013. 111(1): 44-52.

17 Moisseiev E, Regenbogen M, Bartfeld Y, Barak A. Evaluation of pain in intravitreal bevacizumab injections. Curr Eye Res 2012; 37: 813-817.

18 Woods CA, Cumming B. The impact of test medium on use of visual analogue scales. Eye Contact Lens 2009; 35: 6-10.

19 Johnson C. Measuring pain. Visual analog scale versus numeric pain scale: what is the difference? J Chiropr Med 2005; 4: 43-44

20 Breivik EK, Bjornson GA, Skovlund E. A comparison of pain rating scales by sampling from clinical data. Clin J Pain 2000; 16: 22-28.

21 Salo D, Eget D, Lavery RF, Garner L, Bernstein S, Tandon K. Can patients accurately read a visual analog pain scale? Am J Emerg Med 2003; 21: 515-519 
22 Jacobi PC, Dietlein TS, Bacobi FK. A comparative study of topical vs retrobulbar anesthesia in complicated cataract surgery. Arch Ophthalmol 2000; 118: 1037-1043.

23 Crandall AS, Zabriskie NA, Patel BC, Burns TA, Mamalis N, Malmquist-Carter LA et al. A comparison of patient comfort during cataract surgery with topical anesthesia and intracameral lidocaine. Ophthalmology 1999; 106: 60-66.

24 Chiambaretta F, Creuzot-Garcher C, Pilon F, Pouliquen P, Rebika H, Dubray C et al. Ocular tolerance of a new formulation of nonpreserved diclofenac. J Fr Ophthalmol 2004; 27: 739-744.

25 Moisseiev E, Varssano D. Comparison of ocular tolerability between preserved and preservative-free diclofenac sodium drops. J Ocul Pharmacol Ther 2011; 27: 333-337.

26 Yau GL, Jackman CS, Hooper PL, Sheidow TG. Intravitreal injection anesthesia-comparison of different topical agents: a prospective randomized controlled trial. Am J Ophthalmol 2011; 151: 333-337.

27 Cintra LP, Lucena LR, Da Silva JA, Costa RA, Scott IU, Jorge R. Comparative study of analgesic effectiveness using three different anesthetic techniques for intravitreal injection of bevacizumab. Ophthalmic Surg Lasers Imaging 2009; 40: 13-18.

28 LaHood BR, Sherwood D, Suter A. Comparative assessment of the effectiveness of anaesthesia for intravitreal bevacizumab injection. Clin Exp Ophthalmol 2011; 39: 184-185.

29 Heimann $\mathrm{H}$. Intravitreal injections: techniques and sequelae. In: Holz FG, Spaide RF eds Medical Retina (Essentials in Ophthalmology) Chapter 51st ed. SpringerVerlag Berlin Heidelberg: Berlin, 2007, pp 67-87.
30 Sanabria MR, Montero JA, Losada MV, Fernandez-Munoz M, Galindo A, Fernandez I et al. Ocular pain after intravitreal injection. Curr Eye Res 2013; 38: 278-282.

31 Gregori NZ, Weiss MJ, Goldhardt R, Schiffman JC, Vega E, Mattis CA et al. Randomized clinical trial of two anesthetic techniques for intravitreal injections: $4 \%$ liquid lidocaine on cotton swabs versus 3.5\% lidocaine gel. Expert Opin Drug Deliv 2012; 9: 735-741.

32 Davis MJ, Pollack JS, Shott S. Comparison of topical anesthetics for intravitreal injections: a randomized clinical trial. Retina 2012; 32: 701-705.

33 Blaha GR, Tilton EP, Barouch FC, Marx JL. Randomized trial of anesthetic methods for intravitreal injections. Retina 2011; 31: 535-539.

34 Kozak I, Cheng L, Freeman WR. Lidocaine gel anesthesia for intravitreal drug administration. Retina 2005; 25 : 994-998.

35 Rifkin L, Schaal S. Shortening ocular pain duration following intravitreal injections. Eur J Ophthalmol 2012; 22: 1008-1012.

36 Rifkin L, Schaal S. Factors affecting patients' pain intensity during in office intravitreal injection procedure. Retina 2012; 32: 696-700.

37 Rodrigues EB, Grumann A, Penha FM, Shiroma H, Rossi E, Meyer $\mathrm{CH}$ et al. Effect of needle type and injection technique on pain level and vitreal reflux in intravitreal injection. J Ocul Pharmacol Ther 2011; 27: 197-203.

38 Knecht PB, Michels S, Sturm V, Bosch MM, Menke MN. Tunnelled versus straight intravitreal injection: intraocular pressure changes, vitreous reflux, and patient discomfort. Retina 2009; 29: 1175-1181. 